\title{
Simulation of Elastic Rupture in Extension of Entangled Monodisperse Polymer Melts
}

\author{
Lyhne, Anders; Rasmussen, Henrik K.; Hassager, Ole
}

Published in:

Physical Review Letters

Link to article, DOI:

10.1103/PhysRevLett.102.138301

Publication date:

2009

Document Version

Publisher's PDF, also known as Version of record

Link back to DTU Orbit

Citation (APA):

Lyhne, A., Rasmussen, H. K., \& Hassager, O. (2009). Simulation of Elastic Rupture in Extension of Entangled Monodisperse Polymer Melts. Physical Review Letters, 102(13), 138301.

https://doi.org/10.1103/PhysRevLett.102.138301

\section{General rights}

Copyright and moral rights for the publications made accessible in the public portal are retained by the authors and/or other copyright owners and it is a condition of accessing publications that users recognise and abide by the legal requirements associated with these rights.

- Users may download and print one copy of any publication from the public portal for the purpose of private study or research.

- You may not further distribute the material or use it for any profit-making activity or commercial gain

- You may freely distribute the URL identifying the publication in the public portal

If you believe that this document breaches copyright please contact us providing details, and we will remove access to the work immediately and investigate your claim. 


\title{
Simulation of Elastic Rupture in Extension of Entangled Monodisperse Polymer Melts
}

\author{
Anders Lyhne, ${ }^{1}$ Henrik Koblitz Rasmussen, ${ }^{2}$ and Ole Hassager ${ }^{1}$ * \\ ${ }^{1}$ Department of Chemical and Biochemical Engineering, Danish Polymer Center, Technical University of Denmark, \\ 2800 Kgs. Lyngby, Denmark \\ ${ }^{2}$ Department of Mechanical Engineering, Technical University of Denmark, 2800 Kgs. Lyngby, Denmark
}

(Received 12 January 2009; published 2 April 2009)

\begin{abstract}
We use a Lagrangian finite element method to simulate experiments on continued and interrupted extensions of a highly entangled monodisperse polymer melt. It is demonstrated that delayed sample necking or rupture may be explained in terms of the original Doi-Edwards model augmented by the mechanism of chain stretching alone.
\end{abstract}

DOI: 10.1103/PhysRevLett.102.138301

PACS numbers: 83.80.Sg, 83.10.Kn, 83.50.Jf

In a pioneering development, Doi and Edwards [1] constructed a model for the dynamics of highly entangled monodisperse polymer melts. The model was based on the idea that a given polymer chain is moving by reptation [2] in a tube made up effectively by the surrounding chains. Based on this model concept, they derived scalings for the dependence of viscosity and diffusivity on molar mass. Moreover they developed an integral constitutive equation [3] for the stress as a function of kinematic history. While the original model is known to have many limitations, the basic idea of a confining tube is still utilized extensively in the modeling of entangled polymer systems. However, recently Wang and co-workers [4] have published intriguing experiments on entangled polymer melts and corresponding modeling ideas that they claim are needed to explain their data. These ideas depart fundamentally from the tube model and the established methods of polymer kinetic theory [5]. In view of the remarkable advances in our understanding of polymer dynamics over the last $25 \mathrm{yr}$, it seems reasonable to reconsider the experiments by Wang and co-workers in the framework developed upon the original DE model.

Wang considered a series of four monodisperse styrenebutadiene (SBR) plus one polyisoprene (PIP) polymer melts. Samples prepared from the melts were extended by rolling them on two counter rotating cylinders (a SER fixture [6]) at a rate considerably faster than the inverse reptation time of the melt. Given an initial length $L_{0}$ and a current length $L(t)$, the Hencky strain at time $t$ is defined by $\epsilon=\ln L(t) / L_{0}$. In phase 1 of the experiments, the angular velocity of the cylinders was kept constant. At a given preset strain, the rotation of the cylinders was interrupted and the ensuing stress relaxation in the samples was observed in a second phase of the experiment. In this letter we consider the following key observations on the $250 \mathrm{~K} \mathrm{SBR}$ rubber (none of which can be explained by the original DE model): (i) In phase 1 (continuous extension), the samples could be extended to about $\epsilon \approx 2$ without necking. (ii) In phase 2 (interrupted extension), samples extended initially to $\epsilon=0.6$ would remain intact, while samples extended to $\epsilon=0.8$ and higher would suddenly neck after a period considerably shorter than the terminal relaxation time. (iii) The time to the onset of necking decreases with increasing initial imposed Hencky strain. The DE equation for the viscoelastic stress tensor, $\boldsymbol{\sigma}$, may be formulated in the form [3]

$$
\boldsymbol{\sigma}(t)=\int_{-\infty}^{t} M\left(t-t^{\prime}\right) \mathbf{Q}\left(\mathbf{x}, t, t^{\prime}\right) d t^{\prime},
$$

where $\mathbf{Q}$ is the Doi-Edwards strain tensor and $M$, the memory function accounting for the reptation of the polymer chain out of deformed tube sections. In order to simplify computations we approximate the Doi-Edwards strain tensor as follows,

$$
\mathbf{Q}_{A}\left(\mathbf{x}, t, t^{\prime}\right)=\frac{\gamma_{[0]}\left(\mathbf{x}, t, t^{\prime}\right)}{\operatorname{tr}\left[\mathbf{B}\left(\mathbf{x}, t, t^{\prime}\right)\right]}=\frac{\gamma_{[0]}\left(\mathbf{x}, t, t^{\prime}\right)}{I_{1}\left(\mathbf{x}, t, t^{\prime}\right)},
$$

where $\mathbf{B}$ is the Finger strain tensor, $\gamma_{[0]}=\mathbf{I}-\mathbf{B}$ is the corresponding finite strain tensor that reduces to the infinitesimal strain tensor for small deformations and $I_{1}$ is the trace of the Finger tensor [3]. In fast extensional flow, the DE model predicts an upper limit to the tensile stress so the total force exhibits a maximum whereby the filament becomes unstable [7]. In the original DE model this critical strain is 0.86 , while for the approximation in (2) the critical Hencky strain is 0.63 . Hence the DE equation cannot describe observation 1 as remarked in [4]. The molecular origin of the limiting stress is the assumption of instantaneous chain retraction so that the stress is due to orientation only. However, it was recognized early that chain stretching will occur at deformation rates faster than the inverse Rouse time [8,9]. The concept of chain stretching is now standard in reptation based models [10-12]. We use the formulation due to McLeish and Larson [13] for inspiration:

$$
\frac{\partial}{\partial t} \lambda=\lambda \boldsymbol{\kappa}: \mathbf{S}-\frac{1}{\tau_{s}}(\lambda-1)
$$

Here $\lambda$ is the chain stretch relative to equilibrium, $\boldsymbol{\kappa}$ the velocity gradient, $\mathbf{S}$ a nondimensional structure tensor and 
$\tau_{s}$ a time constant for stretch relaxation. In the limit of rapid stretching $\mathbf{S}=\mathbf{B} / I_{1}$ and (3) has the solution [7]:

$$
\lambda^{2}=I_{1} / 3
$$

On the other hand, if we neglect the stress growth term, we obtain the following solution

$$
\lambda^{2}=\left[1+\left(\lambda_{0}-1\right) e^{-t / \tau_{s}}\right]^{2},
$$

where $\lambda_{0}$ is an initial value, which can be approximated by the rapid stretch result of (4). A simple expression that combines the two expressions is

$$
\lambda^{2}\left(\mathbf{x}, t, t^{\prime}\right)=\left[1+\left(\sqrt{I_{1} / 3}-1\right) e^{-\left(t-t^{\prime}\right) / \tau_{s}}\right]^{2}
$$

These considerations suggest that chain stretching may be approximately incorporated into the DE model by the following integral constitutive equation

$$
\sigma(\mathbf{x}, t)=\int_{-\infty}^{t} \lambda^{2}\left(\mathbf{x}, t, t^{\prime}\right) M\left(t-t^{\prime}\right) \mathbf{Q}_{A}\left(\mathbf{x}, t, t^{\prime}\right) d t^{\prime},
$$

where $\lambda$ is given by (6) and $\mathbf{Q}_{A}$ is given by (2). In contrast to the original formulation in which a single stretch factor applies to the entire chain, we include the stretch factor inside the integral [14,15], whereby the stretch factor of a given chain segment depends on the strain since the creation of that segment. The memory function we use is derived from the BSW spectrum for linear viscoelasticity [16],

$$
M(s)=n_{e} G_{N}^{0} \int_{0}^{\lambda_{m}}\left(\frac{\lambda}{\lambda_{m}}\right)^{n_{e}} \frac{e^{-s / \lambda}}{\lambda^{2}} d \lambda,
$$

where $G_{N}^{0}$ is the plateau modulus, $n_{e}$ a nondimensional parameter and $\lambda_{m}$ a time constant. The BSW spectrum is intended for polymers of uniform length such as the monodisperse SBR considered here.

Our final constitutive equation [Eqs. (2) and (6)-(8)] contains $3 \mathrm{BSW}$ parameters associated with the linear viscoelastic behavior $\left(n_{e}, G_{N}^{0}, \lambda_{m}\right)$ and one time constant for nonlinear relaxation $\left(\tau_{s}\right)$. We have fitted the BSW parameters to the loss and storage moduli for the $100 \mathrm{~K}$ material [17] by a least squares method. The resulting parameters are shown in Table I while the quality of the fit may be evaluated from Fig. 1. There is a clear deviation in the loss modulus at high frequencies. This is because we have omitted the glassy part of the spectrum. The glassy

TABLE I. Parameters for $100 \mathrm{~K} \mathrm{SBR}$ (fitted to data by [17]) and the $250 \mathrm{~K} \mathrm{SBR}$.

\begin{tabular}{lcc}
\hline \hline & $100 \mathrm{~K} \mathrm{SBR}$ & $250 \mathrm{~K} \mathrm{SBR}$ \\
\hline$n_{e}$ & 0.261 & 0.261 \\
$G_{N}^{0}[\mathrm{MPa}]$ & 0.652 & 0.652 \\
$\lambda_{m}[\mathrm{~s}]$ & 57.95 & 1300 \\
$\tau_{s}[\mathrm{~s}]$ & $\cdots$ & 5.7 \\
\hline \hline
\end{tabular}

modes are expected to be negligible at the extension rates considered here. Table I also contains the parameters for the $250 \mathrm{~K} \mathrm{SBR}$ used here obtained as follows: The parameters $n_{e}$ (related to the slope of $G^{\prime \prime}$ ) and $G_{N}^{0}$ are assumed independent of molar mass while $\lambda_{m}$ is obtained by scaling from the $100 \mathrm{~K}$ value. In Doi-Edwards terms our parameters for $250 \mathrm{~K}$ correspond to a reptation time $\tau_{d} \approx \lambda_{m}(1+$ $\left.n_{e}\right) /\left(2+n_{e}\right)=725 \mathrm{~s}$ and an entanglement molar mass $4 \rho R T / 5 G_{N}^{0} \approx 3000 \mathrm{~kg} / \mathrm{mol}$. Hence the sample has about $Z=83$ entanglements and a Rouse time $\tau_{R} \approx \tau_{d} / 3 Z \approx$ $2.9 \mathrm{~s}$. The stretch relaxation time constant, $\tau_{s}$ was fitted to the rupture data [4] around $\epsilon_{N}=1.5$ but it differs only by a factor 2 from the Rouse time.

We simulated the SER experiment by a 2D planar finite element analysis utilizing a Lagrangian kinematic description. The flow problem is then an initial value problem in which the particle positions are treated as dependent variables for time $t>0$. This is a convenient way of dealing with the free surface and the viscoelastic memory integral constitutive equation. The filament is discretised into a two dimensional grid consisting of quadratic triangular elements. The elements are connected in a structured manner and hereafter the coordinates are transformed to create high and low resolution areas (Fig. 2). Each element is second order in space and zeroth order in pressure, meaning that there will be 12 displacement variables per element and a single element pressure.

The approximated variables are interpolated by the shape functions as

$$
\hat{x}_{i}(t)=\sum_{n=1}^{N} \phi_{n} x_{i, n}(t) \quad \text { and } \quad \hat{p}(t)=\sum_{m=1}^{M} \psi_{m} p_{m}(t)
$$

$\phi$ and $\psi$ are the displacement and pressure shape func-

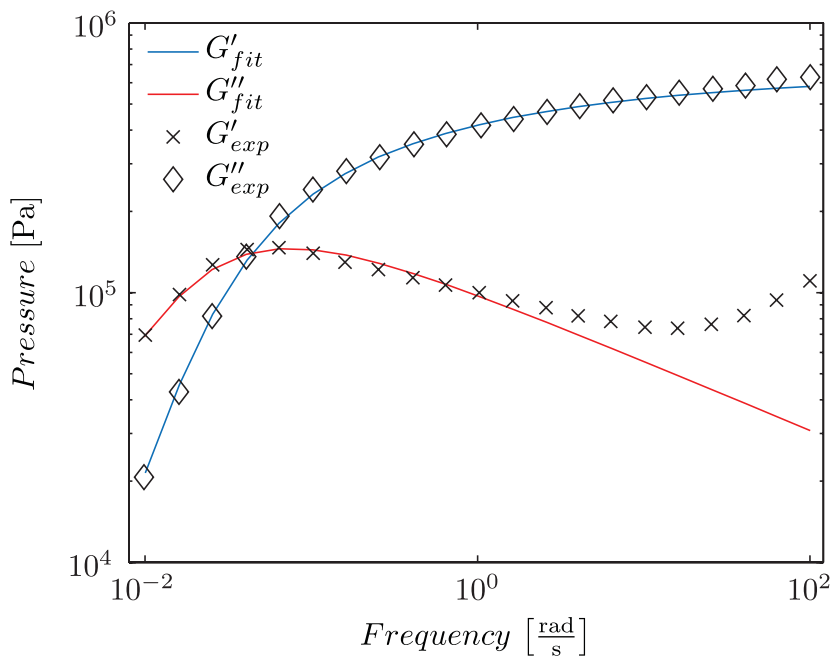

FIG. 1 (color). Data from ([17], Fig. 1) fitted to the BSW spectrum. Only the 12 lower frequency data points were included in the fit, since the glassy part of the BSW model was omitted. 


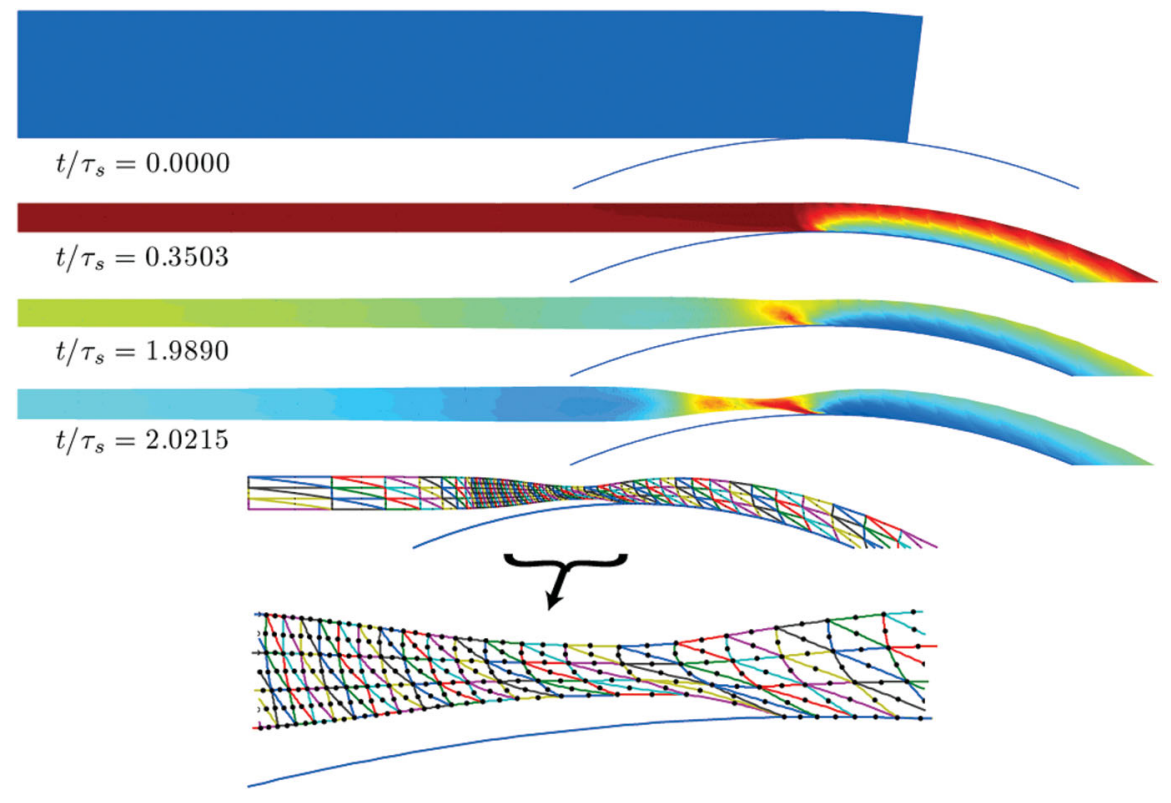

FIG. 2 (color). The sequence to the left shows a typical necking of the sample during relaxation. The top figure shows the filament prior to deformation. The second is immediately after cessation of extension. The next two figures show the ongoing filament necking where $\epsilon_{N}=1.597$ and $\dot{\epsilon}=0.8 \mathrm{~s}^{-1}$. The coloring is a measure of relative internal pressure $($ red $=$ high, blue $=$ low). The two bottom figures show a zoom-in on the necking zone-notice the smooth element to element transition except at the contact point.

tions, respectively, and $x_{i, n}$ and $p_{m}$ are vectors of corresponding nodal values.

The equations of conservation of mass and momentum are formulated in a weak Galerkin formulation whereby approximate Lagrangian coordinates $\hat{x}_{n}(t)$ and $\hat{p}_{m}(t)$ are obtained as function of time [18].

We simulate a SER fixture consisting of two cylinders of radius $R(5.155 \mathrm{~mm})$ with axes separated by a length $2 L_{0}$ $(12.72 \mathrm{~mm})$. The plane $x=0$ defines the symmetry plane for the two cylinders and we consider the domain $x>0$. The initial contact between the cylinder and sample occurs at $x=L_{0}$ while the initial sample thickness is $W_{0}(1 \mathrm{~mm})$. Figure 2 top shows a typical initial condition for a sample. For $t>0$ the two drums are rotated at constant angular velocity $\Omega$ for a predefined time interval $t_{0}$. Then the rotation is halted and the relaxation of the material is observed. The imposed nominal Hencky strain rate during rotation $\dot{\epsilon}_{N}$ and the nominal overall Hencky strain imposed $\epsilon_{N}$ are defined by

$$
\dot{\epsilon}_{N}=\frac{R \Omega}{L_{0}} \quad \text { and } \quad \epsilon_{N}=\dot{\epsilon}_{N} t_{0},
$$

where $2 \pi / \Omega$ would be the period of rotation.

The point of contact between the sample and the cylinder is a crucial part of the problem for $t>0$. To ensure high accuracy in the calculation of this, the exact time of the first contact of a surface node on the cylinders is included as a dependent variable in a computation. This method ensures a near exact numerical match of the real boundary condition. A series of simulations have been performed for overall imposed Hencky strain $\epsilon_{N} \in[0.6 ; 1.9]$. Without the inclusion of the molecular stretch factor (i.e. $\lambda=1$ ) the solution can not be continued beyond an imposed Hencky strain of about 0.63 as expected. Hence the inclu- sion of chain stretching is sufficient to explain observation 1 in [4].

The sequence in Fig. 2 shows a simulated sample deformation in an experiment including the initial sample $\left(t / \tau_{s}=0\right)$, the sample at end of extension $\left(t / \tau_{s}=\right.$ $0.3503)$, and two states $\left(t / \tau_{s}=1.9890\right.$ and 2.0215) during a rapid necking phase that occurs after the duration of about two stretch relaxation times. The necking occurs close to the attachment point and seems to propagate from the inhomogeneous stress condition at the cylinders. The bottom part of Fig. 2 presents a magnification around the area of necking.

Even though the rheometer documentation assumes a uniform Hencky strain equal to $\epsilon_{N}$ along the entire filament, our simulations show a different picture with variations in strain along the sample. We need a method for calculating local values of true strains. As an approximation the sample thickness is used as a measure of true strain. With an initial sample thickness of $W_{0}$ and the thickness at an arbitrarily deformed state $W$, the local Hencky strain is

$$
\epsilon_{H}=\ln \left(\frac{W_{0}}{W}\right)
$$

Here it was used that in planar flow the sample thickness and length are inversely proportional. We use (11) to determine the local strain at the thinnest place of the filament. By approximating the time derivative we have obtained the local strain rate at the neck. These are shown in nondimensional form in Fig. 3 right as a function of the time since the rotation was stopped for seven imposed initial strains. For $\epsilon_{N}=0.60$ (not shown) the sample relaxes without necking. But for $\epsilon_{N} \in[0.804 ; 1.597]$ the strain rate diverges at definite times in agreement with experimental observation 2 . This divergence defines the 

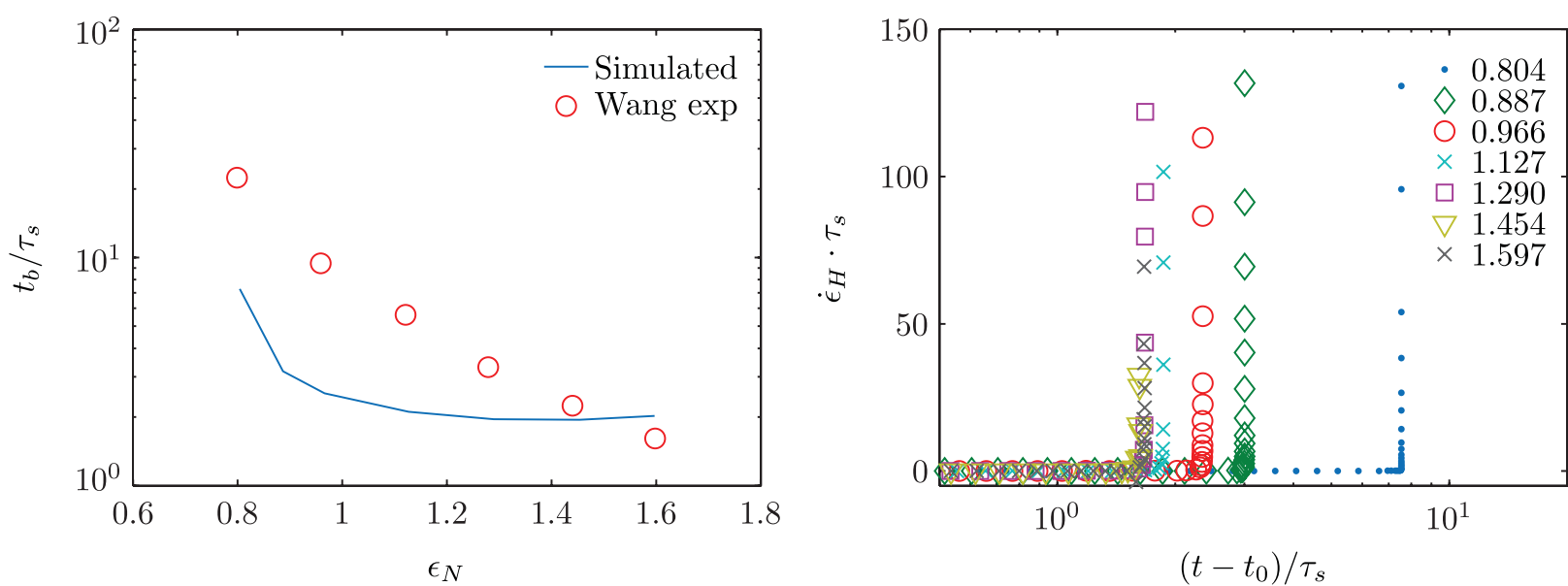

FIG. 3 (color). Simulation of $250 \mathrm{k} \mathrm{SBR}$ filament necking after interrupted extension. Right: Local strain rate at the thinnest place as function of time after imposition of strains 0.804-1.597. Left: Time to necking as function of imposed Hencky strain compared to data by [4]. No delayed necking is observed after imposed Hencky strains $\leq 0.60$.

time to neck, $t_{b}$, since the start of the rotation of the cylinders. The values of $t_{b}$ are shown in Fig. 3 left as function of the imposed strain together with experimental values by [4]. It appears that $t_{b}$ decreases with $\epsilon_{N}$ in qualitative agreement with observation 3 but our simulations show a more abrupt dependence on $\epsilon_{N}$ than the exponential decrease observed by [4], probably because the ansatz for stretch relaxation in (6) with a single nonlinear relaxation time is too simple to describe the nonlinear stretch relaxation process.

We conclude that it is possible to simulate the three observations by [4] and that the observations can be understood in terms of the DE model with chain stretching included. The initial sample stability beyond the Considere limit is due to chain stretching. Conversely the delayed rupture is a consequence of chain stretch relaxation. Once the stress is carried by molecular orientation alone, the sample can no longer remain intact. The time constant for the nonlinear stretch relaxation agrees with our estimate of the Rouse time within expected accuracy in rheological data and model approximations. The simulations also point to the need for improved nonlinear modeling of molecular stretching and relaxation. For this purpose delayed sample rupture provides for rather indirect information whereas recent controlled rheological measurements [15,19] allow for more direct interpretation.

The work was supported by the Danish Technical Research Council (grant 26-04-0074). The authors thank G. H. McKinley for useful comments.

*oh@kt.dtu.dk

Corresponding author.

[1] M. Doi and S.F. Edwards, The Theory of Polymer Dynamics (Clarendon Press, Oxford, 1986).
[2] P. G. de Gennes, Scaling Concepts in Polymer Physics (Cornell University Press, Ithaca, New York, 1979).

[3] R. B. Bird, R. C. Armstrong, and O. Hassager, Dynamics of Polymer Liquids: Fluid Mechanics (John Wiley \& Sons, New York, 1987), Vol. I, 2nd ed.

[4] Y. Wang, P. Boukany, S. Q. Wang, and X. Wang, Phys. Rev. Lett. 99, 237801 (2007).

[5] R.B. Bird, C.F. Curtiss, R.C. Armstrong, and O. Hassager, Dynamics of Polymer Liquids: Kinetic Theory (John Wiley \& Sons, New York, 1987), Vol. II, 2nd ed.

[6] M. L. Sentmanat, Rheol. Acta 43, 657 (2004).

[7] G. H. McKinley and O. Hassager, J. Rheol. (N.Y.) 43, 1195 (1999).

[8] G. Marrucci and N. Grizzuti, Gazz. Chim. Ital. 118, 179 (1988).

[9] D.S. Pearson, A.D. Kiss, L. J. Fetters, and M. Doi, J. Rheol. (N.Y.) 33, 517 (1989).

[10] J. Fang, M. Kröger, and H. C. Öttinger, J. Rheol. (N.Y.) 44, 1293 (2000)

[11] D. W. Mead, R. G. Larson, and M. Doi, Macromolecules 31, 7895 (1998).

[12] J. Schieber, J. Neergaard, and S. Gupta, J. Rheol. (N.Y.) 47, 213 (2003).

[13] T. C. B. McLeish and R. G. Larson, J. Rheol. (N.Y.) 42, 81 (1998).

[14] M. H. Wagner, V.H. Rolon-Garrido, J. K. Nielsen, H. K. Rasmussen, and O. Hassager, J. Rheol. (N.Y.) 52, 67 (2008).

[15] J. K. Nielsen and H. Rasmussen, J. Non-Newtonian Fluid Mech. 155, 15 (2008).

[16] M. Baumgaertel, A. Schausberger, and H. Winter, Rheol. Acta 29, 400 (1990).

[17] Y. Wang and S.-Q. Wang, J. Rheol. (N.Y.) 52, 1275 (2008).

[18] J. M. R. Marin and H. K. Rasmussen, J. Non-Newtonian Fluid Mech. 156, 177 (2009).

[19] J. K. Nielsen, H. K. Rasmussen, and O. Hassager, J. Rheol. (N.Y.) 52, 885 (2008). 